\title{
O Blockchain como meio de prova no Direito Processual Civil Brasileiro
}

\section{Blockchain as Evidence in Brazilian Civil Procedural Law}

\author{
Marcelo Pereira de Almeida ${ }^{1}$ \\ Diogo de Castro Ferreira ${ }^{2}$
}

\begin{abstract}
RESUMO
O presente artigo aborda a possibilidade de utilização do blockchain como meio de prova no processo civil brasileiro. Para isso, passa pela conceituação da tecnologia, por uma análise jurídica da possibilidade de utilização da mesma como meio de prova e, por fim, por um levantamento de como essa matéria vem sendo tratada pelo Poder Judiciário brasileiro. Partese de uma metodologia exploratória, uma vez que se busca investigar a materialização de um fenômeno tecnológico e seus desdobramentos no âmbito do processo civil. Adota-se também a metodologia quantitativa, fazendo-se uso da busca booleana, para se chegar a uma avaliação preliminar acerca da jurisprudência que envolve a matéria.
\end{abstract}

\section{PALAVRAS-CHAVE:}

Blockchain; Meio de prova; Processo Civil

\begin{abstract}
This article discusses the possibility of using blockchain as evidence in the Brazilian civil process. For this, it involves the conception of this technology, a legal analysis of the possibility of using it as evidence and, finally, a survey of how this topic has been treated by the Brazilian Judiciary. The study is based on an exploratory methodology, as it seeks to research the materialization of a technological phenomenon and its consequences within the scope of civil proceedings. A quantitative methodology is also adopted, using a Boolean search, to obtain a preliminary assessment of the jurisprudence that involves a subject.
\end{abstract}

\footnotetext{
1 Pós-Doutor em Direito Processual pela UERJ. Pós- Doutorando em Direito pela Universidade de Burgos (Espanha). Doutor em Ciências Jurídicas e Sociais pela UFF. Professor Adjunto de Direito Processual da UFF, Professor de Direito Processual Civil da Escola da Magistratura do Estado do Rio de Janeiro. Professor Permanente do PPGD (mestrado) da UCP. Coordenador da Pós-graduação em Direito Civil e Processual Civil do Unilasalle/RJ; Coordenador adjunto do Curso de Direito do Unilasalle/RJ; Professor de Teoria Geral do Processo e Direito Processual do Unilasalle/RJ. Membro do Instituto Brasileiro de Direito Processual Civil (IBDP), da Associação Brasileira de Direito Processual (ABDPro) e do Instituto Carioca de Processo Civil - ICPC. Advogado. ORCID: https://orcid.org/0000-0001-7168-097X?lang=pt. marcelo.almeida@ucp.br.

2 Mestrando em Direito pela Universidade Católica de Petrópolis. Possui MBA em Direito Tributário na Fundação Getúlio Vargas de Juiz de Fora. Bolsista CAPES. Vice-presidente e sócio fundador do Instituto de Estudos Tributários e de Finanças Públicas de Juiz de Fora e Região. Vice-presidente da Comissão de Direito Tributário da OAB da subseção de Juiz de Fora - MG. Membro do Conselho Editorial da Pembroke Collins. Membro do CAED-Jus. Membro do Conselho Editorial da Pembroke Collins. Advogado.
} 


\section{KEYWORDS:}

Blockchain; Evidence; Civil Procedure

\section{INTRODUÇÃO}

A tecnologia blockchain vem ganhando espaço em diversas áreas da vida humana. Sua capacidade de resolver determinados problemas e apresentar novas soluções tecnológicas a torna disruptiva, o que tem levado ao aumento de artigos científicos voltados à pesquisa dos possíveis usos dessa tecnologia, que vão muito além das conhecidas criptomoedas, como, por exemplo, o Bitcoin (FERREIRA et al., 2017; CASTAÑEDA-AYARZA et al., 2019).

São incontáveis as possibilidades de aplicação da tecnologia blockchain e, consequentemente, as questões que surgem dessas aplicações. Ela pode ser aplicada, por exemplo, aos smart contracts (MOREIRA, 2019), usada para a finalidade de lavagem de dinheiro (BOTTINO e TELLES, 2018), impõe uma série de desafios regulatórios e fiscais (FERREIRA, 2018), pode ser utilizada na logística reversa (LIMA et al., 2018; SILVA, et al., 2019), apresentar soluções para cartórios (SALLES, 2019) e até mesmo para a área da saúde (MONTEIL, 2019).

O objetivo desse artigo é examinar juridicamente um dos possíveis usos dessa tecnologia, que consiste em servir como meio de prova, sob a ótica do direito processual civil. A relevância do presente artigo se justifica pela novidade do tema, ainda pouco explorado em artigos científicos e até mesmo na jurisprudência, conforme se verá.

Parte-se de um problema fundamental: a tecnologia blockchain é compatível com os meios de prova admitidos no direito processual civil brasileiro? A hipótese aqui apresentada é a de que há compatibilidade e que, além disso, o blockchain pode ser considerado um meio idôneo de prova. Para realizar essa demonstração adota-se a metodologia exploratória, uma vez que se busca investigar a materialização de um fenômeno tecnológico e seus desdobramentos no âmbito do processo civil. Adota-se também a metodologia quantitativa, fazendo-se uso da 
busca booleana, para se chegar a uma avaliação preliminar do estado da arte da jurisprudência que envolve a matéria.

Na segunda seção, serão abordados aspectos conceituais, para que o leitor consiga compreender os principais aspectos técnicos que definem essa tecnologia e, a partir disso, delimitar o seu alcance prático na solução de problemas do mundo real.

Já na terceira seção, será explicado como essa tecnologia pode servir para conferir integridade a determinados dados e, por isso, ser utilizada como meio idôneo de prova no âmbito do direito processual civil.

Por fim, na quarta seção, será abordado os resultados de uma busca booleana com a palavra-chave "blockchain" na seção de jurisprudência do site Jusbrasil. Será realizada a busca completa da plataforma que abrange STJ, STF, TST, TSE, TCU, TNU, CNJ, STM, Tribunais de Justiça, Tribunais Regionais Federais, Tribunais Regionais do Trabalho, Tribunais Regionais Eleitorais, Tribunais de Contas dos Estados e Tribunal de Justiça Militar. Os resultados serão quantificados e divididos por órgãos julgadores. Por fim, será traçado um panorama geral dos julgados encontrados nos dois primeiros resultados e uma análise pormenorizada de alguns dos julgados, com base em uma análise subjetiva de relevância e aproximação temática.

\section{ASPECTOS CONCEITUAIS}

Em uma rasa e preliminar definição, o blockchain trata-se uma tecnologia de gerenciamento de dados pautada por uma arquitetura descentralizada e criptografada. Quando o conceito é trazido dessa forma, fica parecendo se tratar de algo simples e, por esse motivo, é necessário um certo grau de aprofundamento conceitual para entender o que torna essa tecnologia tão disruptiva e especial.

Traduzindo o termo literalmente, blockchain significa cadeia de blocos. A tecnologia em questão surgiu em 2008, inaugurada por um manuscrito de Satoshi Nakamoto, denominado

"Bitcoin: A Peer-to-Peer Eletronic Cash System". Satoshi Nakamoto trata-se de um 
pseudônimo utilizado por aquele ou aqueles que inauguraram as bases teóricas do blockchain (NAKAMOTO, 2008).

Nesse sentido, para se entender a primeira característica fundamental dessa tecnologia, é preciso subdividir as arquiteturas dos softwares em duas categorias: a primeira composta pelos centralizados e a segunda pelos distribuídos, ou descentralizados (TANENBAUM e STEEN, 2007).

Nos softwares centralizados, a arquitetura de validação de dados e trocas de informações são realizadas por uma espécie de componente central. É o que ocorre, por exemplo, em um banco ou em um cartório. Quando uma pessoa transfere certo montante em dinheiro para outra pessoa, a transferência do valor passa pela validação da instituição bancária, que se vale de um software centralizado, onde apenas um núcleo detém aquelas informações. O mesmo ocorre com a transferência de um bem imóvel. O cartório caso se valha de algum software onde essa troca de informações é validada, também se valerá de uma arquitetura centralizada.

Já nos softwares descentralizados, ou distribuídos, como é o caso do blockchain, os componentes se conectam entre eles sem que haja um elemento central responsável pelo gerenciamento dos dados. Existem quatro principais vantagens de um software de arquitetura distribuída: mais capacidade de processamento, redução de custos, mais confiabilidade e capacidade de expandir-se naturalmente (DRESCHER, 2017).

A superioridade de processamento se explica pelo fato de que em um sistema distribuído a capacidade de processamento se dá através da combinação do rendimento dos vários computadores empenhados na ação de processamento, o que, naturalmente, tende a superar um computador individual, ou mesmo um conjunto deles realizando essas tarefas em um ambiente centralizado.

O elemento da redução de custos está atrelado ao fato de que a manutenção de um supercomputador costuma ser mais cara do que a de diversos computadores convencionais distribuídos em uma rede descentralizada.

Já a confiabilidade em nível mais elevado que o de sistemas centralizados pode ser explicado pelos muitos pontos de falha espalhados em um sistema distribuído. Isso possibilita 
que, caso máquinas individuais falhem, o sistema distribuído continuará funcionando, diferentemente de uma falha ocorrida em um sistema centralizado.

Por fim, a característica de maior capacidade de expandir-se naturalmente se dá pelo fato de que toda vez que um computador novo é agregado ao sistema, aumenta-se a capacidade de processamento. Desta forma, a capacidade de processamento de todo o sistema pode ser expandida de maneira incremental.

Entretanto, em um sistema puramente distribuído, apresentam-se cinco principais desvantagens: overhead de coordenação, overhead de comunicação, dependência de redes, mais complexidade nos programas e problemas de segurança (DRESCHER, 2017).

Quando se fala em overhead $^{3}$ de coordenação e comunicação, no primeiro caso, tratase do desafio de coordenação em um sistema distribuído, ou descentralizado, uma vez que essa tarefa deve ser desempenhada pelos próprios computadores que compõem a rede. Como coordenação exige, necessariamente, comunicação, daí se extrai o segundo conceito, uma vez que nesses sistemas a comunicação entre os computadores que fazem parte do sistema apresenta-se também como um desafio.

As redes são o meio pelo qual a coordenação e a comunicação acontecem. Sem elas, não poderia haver comunicação entre os computadores empenhados no sistema, portanto, a dependência das redes é outro ponto frágil que merece destaque. Como os três problemas mencionados anteriormente são extremamente complexos de serem resolvidos, deles decorre, logicamente, uma maior complexidade na programação dos softwares descentralizados.

Por fim, um dos maiores problemas em um sistema puro de software distribuído é o problema da segurança. Esse problema surge em decorrência dos dados críticos que podem ser trocados em uma rede descentralizada, como dados financeiros, por exemplo. Isso cria brecha para que usuários mal-intencionados busquem a interceptação desses dados trocados.

Nesse momento, em que foram explicados os conceitos de sistemas centralizados e sistema distribuídos, bem como as vantagens e desvantagens dos sistemas descentralizados, faz-se uma importante indagação: o blockchain consegue resolver os problemas apontados dos

\footnotetext{
${ }^{3}$ Overhead é um termo que, em português, significa sobrecarga.
} 
sistemas distribuídos? Em resposta provisória, a resposta é sim. O blockchain é uma ferramenta para prover, justamente, a integridade de sistemas descentralizados de softwares.

Para entender como o blockchain oferece as soluções para os principais problemas apresentados pelos sistemas distribuídos é necessário que adentremos no conceito de arquitetura $^{4}$ peer-to-peer (P2P). Sinteticamente, o sistema $\mathrm{P} 2 \mathrm{P}$, ou ponto a ponto, tem como objetivo principal fazer com que cada computador distribuído pela rede seja ao mesmo tempo usuário e disponibilizador de recursos.

A rede peer-to-peer pode ser compreendida como uma rede conectada de computadores onde cada peer, denominação dada a esses computadores, pode atuar tanto como cliente quanto como servidor. Seu nascimento se mostra como uma alternativa à clássica arquitetura clienteservidor, e seus principais objetivos são aumentar a disponibilidade do recurso e aumentar a largura de banda de upload do sistema (ORAM, 2001).

Talvez o melhor exemplo do potencial da tecnologia P2P seja o Napster, programa de computador criado no final da década de noventa por um garoto chamado Shawn Fanning. O programa foi considerado revolucionário, uma vez que possibilitou a criação de redes de troca de arquivos peer-to-peer, o que pareceu a Fanning o instrumento perfeito para o compartilhamento de música digital, em um contexto favorecido pela recém popularização do formato MP3 (SOARES et al., 2008).

A lógica do programa era cada computador da rede cujo objetivo fosse baixar uma música, passasse, a partir do momento em que realizasse o donwload, a ser também um fornecedor daquela música aos demais usuários da rede. Lógica similar ao sistema de Torrents criado, posteriormente, em 2005. O programa Napster foi tão disruptivo em seu tempo que obrigou a indústria da música a se reformular, abrindo precedente para que também a indústria cinematográfica tivesse que seguir o mesmo caminho. Foi o pontapé inicial para tornar obsoletas as fitas cassetes, CDs e DVDs e obrigar que essas indústrias de reformulassem.

Restam explicados os conceitos de sistemas centralizados, sistemas distribuídos e arquitetura peer-to-peer, todas características próprias do blockchain, mas ainda falta um

\footnotetext{
${ }^{4} \mathrm{O}$ termo "arquitetura" empregado no contexto do presente artigo remete à arquitetura de redes de computadores, especificamente a arquitetura peer-to-peer, onde cada um dos nós da rede funciona tanto como cliente quanto como servidor.
} 
elemento para completar a elucidação de como essa tecnologia fornece integridade ao sistema com preservação das informações trocadas na rede. Para isso, é necessário explorar os conceitos de criptografia e, finalmente, o funcionamento e a lógica da chamada cadeia de blocos.

A tecnologia blockchain se compromete a validar a existência ou não da posse de determinados dados, garantindo a integridade e a inviolabilidade dos mesmos. Nesse contexto, um dos principais problemas solucionados por essa tecnologia é o problema do gasto duplo.

Em um sistema distribuído ponto a ponto, todos os computadores são responsáveis pela manutenção e pela ordem dos dados trocados entre os usuários. Como esses dados ficam armazenados, ao mesmo tempo, para todos os usuários? Através de uma espécie de livro-razão único. Isso significa dizer que, em um sistema ponto a ponto distribuído de livros-razão, todos os nós, ou computadores, espalhados pelo sistema possuem um mesmo conjunto de informações inseridas, ou seja, todos possuem o mesmo livro-razão, com os mesmos dados inseridos e na mesma sequência.

O tempo despendido para que todos os computadores passem a ter essa mesma sequência unitária de dados simultaneamente poderia muito bem ser explorada por um usuário mal-intencionado. Suponhamos que esse sistema fosse responsável pela guarda e transferência de dados relativos à propriedade de veículos automotores. Quando um elemento "a" realiza a transferência de um bem imóvel a um elemento " $b$ ", teoricamente, ele poderia valer-se do lapso temporal que essa informação demoraria a constar no livro-razão de todos os computadores da rede para vender esse mesmo automóvel para um elemento "c". Eis o problema do gasto duplo.

Porém, a tecnologia blockchain resolve esse problema diretamente relacionado à integridade do sistema da seguinte forma:

“O Blockchain enquanto 'banco de dados' é atualizado de tempos em tempos constituindo-se, portanto, numa crescente conexão de 'blocos de informação'. Cada bloco é um conjunto contendo diversas informações ocorridas entre os intervalos de atualização da Blockchain. No caso da Blockchain do Bitcoin, estas informações são transações financeiras intersubjetivas.

Neste viés, entende-se a significância metafórica do vocábulo blockchain, de origem inglesa, significando 'corrente de blocos'. Metaforicamente, é como se a Blockchain fosse uma 'corrente' - banco de dados atualizável - de quantidade crescente de 'elos'. Cada 'elo da corrente' seria um 'bloco' contendo diversas informações ocorridas num certo intervalo de tempo.

Quando ocorre uma nova transação de bitcoin, ela é, num certo sentido, 'carimbada' com data e hora e analisada contra o atual Blockchain do Bitcoin visando assegurar a eliminação do problema do gasto duplo. Desta forma, uma nova transação de bitcoin 
será uma, dentre muitas outras, pertencentes a um certo bloco de informações que, após determinado intervalo de tempo, será adicionado à corrente de blocos.

Caso se tente usar um mesmo quantum de bitcoin mais de uma vez, numa duplicidade de transação, tem-se que, devido ao 'carimbo com data e hora', a rede global14 peerto-peer desconsidera a segunda transação e mantém a primeira, conforme a 'carimbação de tempo' respeitando a perfeita ordem de ocorrência cronológica." (COSTA, 2019).

Superado o problema do gasto duplo, o último desafio do blockchain diz respeito à proteção dos dados contra acessos por pessoas não autorizadas:

\footnotetext{
“As transações são verificadas, e o gasto duplo é prevenido, por meio de um uso inteligente da criptografia de chave pública. Tal mecanismo exige que a cada usuário sejam atribuídas duas 'chaves', uma privada, que é mantida em segredo, como uma senha, e outra pública, que pode ser compartilhada com todos. Quando a Maria decide transferir bitcoins ao João, ela cria uma mensagem, chamada de 'transação', que contém a chave pública do João, assinando com sua chave privada. Olhando a chave pública da Maria, qualquer um pode verificar que a transação foi de fato assinada com sua chave privada, sendo, assim, uma troca autêntica, e que João é o novo proprietário dos fundos. A transação - e portanto uma transferência de propriedade dos bitcoins é registrada, carimbada com data e hora e exposta em um 'bloco' do blockchain (o grande banco de dados, ou livro - razão da rede Bitcoin). A criptografia de chave pública garante que todos os computadores na rede tenham um registro constantemente atualizado e verificado de todas as transações dentro da rede Bitcoin, o que impede o gasto duplo e qualquer tipo de fraude" (ULRICH, 2014).
}

Após essa breve exposição conceitual onde foi possível compreender o motivo pelo qual a tecnologia blockchain se mostra como capaz de resolver os principais problemas de integridade de um sistema distribuído de ponta a ponta, inaugura-se a análise de como essa tecnologia pode servir como meio de prova no processo civil brasileiro.

\section{A COMPATIBILIDADE DO BLOCKCHAIN, ENQUANTO MEIO DE PROVA, COM O PROCESSO CIVIL BRASILEIRO}

Primeiramente, é necessário entender como a tecnologia blockchain poderia, tecnicamente, servir como prova no processo civil brasileiro para, em seguida, iniciar uma análise propriamente jurídica.

A época atual tem sido marcada pelo termo fake news, onde conteúdos falsos são facilmente disseminados, principalmente com o advento das redes sociais, como Facebook, Instagram, Whatsapp, dentre outras. Nesse contexto tecnológico, existe um duplo desafio a ser 
transposto, o primeiro diz respeito à veracidade das informações divulgadas e, o segundo, da integridade dos dados virtuais. Os dados virtuais são o instrumento através do qual uma informação, seja ela verdadeira ou falsa, é veiculada na internet.

O blockchain pode ser útil quando o assunto é a integridade desses dados. Exemplo prático da possível utilização da tecnologia é o serviço PACWeb, oferecido pela empresa OriginalMy ${ }^{5}$. Se uma pessoa é vítima de crime contra a honra em uma rede social e resolve realizar um print, que é uma espécie de captura da imagem de uma tela. Essa prova em formato de imagem seria, por si só, capaz de comprovar que aquele conteúdo, de fato, foi postado? A resposta correta provavelmente será não, uma vez que qualquer imagem pode ser modificada em um programa de computador.

A prova documental pode ser conceituada como a representação física que visa corroborar o fato alegado pela parte. Com relação à autenticidade da prova documental, seja ela fotografia, desenho, escritos fiscais ou gravações, considera-se autêntica quando, após apresentada em juízo, não houver impugnação da parte contrária, nos termos do art. 411, inciso III do CPC/15 ${ }^{6}$. (OLIVEIRA e CARDOSO, 2019)

O art. 369 do CPC/15 é bastante flexível com relação ao rol de provas que podem ser utilizados pelas partes no processo ao estabelecer que não há um rol taxativo que limite os meios de provas possíveis de serem empregados no processo civil. ${ }^{7}$ Importante ressaltar que o direito à prova é um desdobramento do contraditório, consectário do devido processo e que, assim, as limitações probatórias devem ser mínimas.

Apesar disso, o art. 428, I e II, do CPC/15 estabelece a cessação da fé do documento particular quando for impugnada sua autenticidade e enquanto não se comprovar sua veracidade, ou, assinado em branco, for impugnado seu conteúdo, por preenchimento abusivo.

Desta forma, percebe-se a fragilidade do meio de prova coletado na forma de print e é nesse contexto que surge o serviço oferecido pela OriginalMy como uma das opções que

\footnotetext{
${ }^{5}$ https://originalmy.com/pacweb

${ }^{6}$ Art. 411. Considera-se autêntico o documento quando:

III - não houver impugnação da parte contra quem foi produzido o documento.

${ }^{7}$ Art. 369. As partes têm o direito de empregar todos os meios legais, bem como os moralmente legítimos, ainda que não especificados neste Código, para provar a verdade dos fatos em que se funda o pedido ou a defesa e influir eficazmente na convicção do juiz.
} 
emprega a tecnologia blockchain para a garantia da integridade dos dados encontrados na internet.

De acordo com o site, ao descrever o serviço PACWeb:

\begin{abstract}
"A PACWeb faz uma cópia completa do conteúdo que está sendo visualizado no seu navegador e gera um relatório comprovando a sua existência. A partir do relatório gerado pelo plugin, é criado um hash, código único e exclusivo, que identifica o seu documento em nosso site" (ORIGINAL MY, 2020).
\end{abstract}

Quando busca explicar o motivo pelo qual a empresa resolveu trabalhar com o blockchain, a empresa afirma que:

"Com a certificação em blockchain, é possível comprovar que a prova coletada não sofreu qualquer modificação, com o conteúdo sendo preservado mesmo que a ação original seja excluída. Ele recebe ainda uma camada adicional de proteção que indica a data e horário exatos em que a prova foi coletada. (ORIGINAL MY, 2020)"

Essa explicação se conecta aos conceitos contidos na seção 2 desse artigo, onde abordou-se que a tecnologia blockchain se pauta por um livro-razão único, formado por uma cadeia inalterada de blocos espalhados por todos os computadores que compõem o sistema. Nesse contexto, é possível provar, de forma segura, que tal informação se fazia presente em um site em certo momento do tempo. Não sendo tecnicamente possível, desta forma, ocultar a prova com a ação de deletá-la da rede e a impugnando posteriormente no âmbito judicial usando como argumento a fragilidade da prova.

Entendidas as possibilidades de aplicação prática do blockchain como meio idôneo de prova, bem como as questões jurídicas que envolvem o tema, passa-se à análise de como a questão vem sendo enfrentada no âmbito do Poder Judiciário.

\title{
4 JULGADOS SOBRE O BLOCKCHAIN COMO MEIO DE PROVA
}

Nesse tópico serão explorados, através de metodologia quantitativa, os resultados de uma busca booleana realizada com a palavra-chave blockchain na aba de jurisprudência do site Jusbrasil (JUSBRASIL, 2020). O objetivo não é esgotar os julgados sobre o tema, mas apenas trazer um esboço de como a matéria vem sendo enfrentada pelos órgãos e tribunais. A busca 
foi realizada de forma completa, abrangendo STJ, STF, TST, TSE, TCU, TNU, CNJ, STM, Tribunais de Justiça, Tribunais Regionais Federais, Tribunais Regionais do Trabalho, Tribunais Regionais Eleitorais, Tribunais de Contas dos Estados e Tribunal de Justiça Militar.

Os resultados foram filtrados por órgãos e tribunais, quantificados e, por fim, através da metodologia exploratória, serão explorados apenas os julgados que mais se aproximam da temática do presente artigo.

Ao separar os resultados por número de julgados contendo a palavra-chave blockchain chegou-se ao seguinte resultado:

\begin{tabular}{|c|c|}
\hline Órgão Julgador & Número de julgados \\
\hline TCU & 17 \\
\hline TJSP & 9 \\
\hline TJDF & 6 \\
\hline STJ & 4 \\
\hline TRT10 & 1 \\
\hline TJMG & 1 \\
\hline TJMS & 1 \\
\hline TCE-PR & 1 \\
\hline TOTAL DE JULGADOS & 40 \\
\hline
\end{tabular}

O órgão julgador que mais menciona a palavra-chave "blockchain" é o TCU, que, assim como o CNJ, trata-se de um órgão administrativo e não judicial. Isso se dá pois, em vários desses julgados, o Tribunal de Contas da União discutiu o potencial do uso da tecnologia blockchain na prestação de contas públicas. Outro tema recorrente é o uso do blockchain para a criação de uma criptomoeda indígena, o que, segundo o julgado, poderia fomentar o desenvolvimento econômico das regiões habitadas pela população indígena.

Outro ponto destacado em alguns dos julgados do TCU é a alteração da Portaria RFB 1.639/2016 pela Portaria RFB 1.788, de 19/11/2018 para permitir uma nova forma de acesso por meio da tecnologia blockchain - ao protocolo de compartilhamento de dados do CPF.

Já no TJSP, observa-se conflitos que, na maior parte dos casos, se referem à imposição de prazo de liquidação às exchanges, que funcionam como uma espécie de corretora de criptomoedas. 
Finalmente, será feita a análise dos julgados que, diretamente, estão atrelados ao tema desse artigo. O primeiro caso diz respeito ao Agravo de Instrumento $\mathrm{n}^{\mathbf{0}}$ 223725377.2018.8.26.0000, que tramitou no TJSP, em que o ex-governador do Goiás, Marconi Perillo, pediu a retirada de conteúdo que considerou falso e ofensivo de redes sociais, como Facebook, Instagram e Twitter.

Uma das discussões ocorridas nesse recurso versa sobre a necessidade ou não de se comunicar terceiros acerca dos requerimentos do agravante, pois havia o receio de que, uma vez comunicados, os mesmos apagassem o conteúdo.

Ao apreciar esse pedido, a Desembargadora Fernanda Gomes Camacho entendeu que não havia necessidade, pois havia sido utilizada a tecnologia blockchain oferecida pela plataforma OriginalMy para validação dos dados inseridos nessas redes sociais e, portanto, uma vez inseridos na cadeia de blocos, a prova estaria cristalizada:

\footnotetext{
"Outrossim, não se justifica a pretensão de abstenção de comunicação de terceiros a respeito dos requerimentos do agravante e dos termos da demanda, inclusive porque o próprio recorrente afirmou que 'a partir do conhecimento dos fatos, o Autor providenciou a preservação de todo o conteúdo via Blockchain, junto à plataforma OriginalMY, hábil a comprovar a veracidade e existência dos conteúdos'.” (BRASIL, 2018)
}

Outro caso que merece destaque é o do Agravo de Instrumento $\mathrm{n}^{\mathrm{o}}$ 141263557.2019.8.12.0000, que tramitou no TJMS. No caso se discutiu o indeferimento do pedido de juntada de prints de conversas do aplicativo Whatsapp e de áudios relativos à negociação de compra e venda do automóvel objeto da demanda. No relatório o Desembargador Relator Dorival Renato Pavan ressalta o seguinte:

\footnotetext{
"Discorre sobre a veracidade das provas, destacando que a apresentação delas foi espontânea e em evidente ausência de má-fé, sendo, ademais, que junta ao processo os certificados de autenticidade com registro Blockchain e suas respectivas Hash's, de modo a comprovar a veracidade do conteúdo." (BRASIL, 2019)
}

Nesse caso a validação das provas via blockchain não foi abordada com a mesma ênfase dada na decisão anterior analisada, sendo esse trecho um pequeno detalhe do julgado em questão, onde a discussão foi travada em um âmbito um tanto quanto alheio a esse fato narrado. Porém, é importante verificar que o uso do blockchain como ferramenta para atestar a 
integridade de dados virtuais começa a ganhar espaço nos embates travados no âmbito do Poder Judiciário.

\section{CONCLUSÃO}

A primeira conclusão que se chega é a de que a tecnologia blockchain pode ter um impacto significativo quando o assunto se refere aos meios de prova empregados no processo civil. O principal motivo é o fato dela conferir integridade a dados digitais, o que gera uma prova dotada de idoneidade. Quando pensamos em um contexto onde boa parte das relações entre as pessoas se dá através da internet, que por vezes se encontra repleta de dados falsos, ou até mesmo injuriosos, que podem desaparecer com a simples exclusão de seus conteúdos, a perspectiva é que essa ferramenta seja cada vez mais utilizada para conferir integridade a esses dados com a finalidade de se formar uma prova processual robusta e válida.

Com relação ao tema abordado na jurisprudência, pode-se concluir que o tema blockchain ainda é pouco abordado no Poder Judiciário e demais órgãos julgadores, visto que o total de resultados encontrados na plataforma Jusbrasil com a palavra-chave "blockchain" foi 40. Entretanto, em dois precedentes pode-se observar que o blockchain já foi utilizado como meio de garantir idoneidade a dados digitais utilizados como prova no âmbito do processo civil, o que demonstra que o objeto abordado nesse artigo já está sendo objeto de apreciação pelo Poder Judiciário.

\section{REFERÊNCIAS BIBLIOGRÁFICAS}

BOTTINO, Thiago; TELLES, Christiana Mariani da Silva. Lavagem de dinheiro, bitcoin e regulação. Revista Brasileira de Ciências Criminais, v. 148, 2018.

BRASIL. Tribunal de Justiça do Estado de São Paulo. Agravo de Instrumento $\mathrm{n}^{\circ}$ 223725377.2018.8.26.0000. Agravante: Marconi Ferreira Perillo Junior. Agravados: Twitter Brasil 
Rede de Informação LTDA e Facebook Serviços Online do Brasil LTDA. Relatora: Desembargadora Fernanda Gomes Camacho. São Paulo, 19 de dezembro de 2018.

BRASIL. Tribunal de Justiça do Estado de Mato Grosso do Sul. Agravo de Instrumento $\mathrm{n}^{\circ}$ 1412635-57.2019.8.12.0000. Agravante: 7 Reis Participação e Empreendimentos - Eireli. Agravados: Mirna Saad Cruz e Ministério Público Estadual. Relator: Desembargador Dorival Renato Pavan. Campo Grande, 9 de outubro de 2019.

CASTAÑEDA-AYARZA, Juan Arturo; NEVES, Cíntia; TEIXEIRA, André Frazão. Pesquisa bibliográfica sobre os estudos científicos relacionados com o bitcoin e a blockchain. Revista Contemporânea de Economia e Gestão, v. 17, n. 3, p. 79, 2019.

COSTA, Felipe Ramos. Os criptoativos: historicidade, natureza jurídica, conceito e problematizações jurídico-econômicas. Monografia (Monografia em direito) - UFJF. Juiz de Fora, p. 17, 2019.

DRESCHER, Daniel. Blockchain básico: uma introdução não técnica em 25 passos. 1. Ed. São Paulo: Novatec Editora, p. 24 - 17, 2017.

FERREIRA, Diogo de Castro. As criptomoedas e osdesafios regulatórios e fiscais. In: CONTRERAS, Edgar Fuentes; FILPO, Klever; LIMA, Monique Falcão. Inovação e produção de direito. Rio de Janeiro. Grupo Multifoco, 2018. p. 297 - 314.

FERREIRA, Juliandson Estanislau; PINTO, Filipe Gutemberg Costa; SANTOS, Simone Cristiane dos. Estudo de mapeamento sistemático sobre as tendências e desafios do blockchain. Revista Eletrônica de Gestão Organizacional, v. 15, Edição Especial, p. 112, 2017.

JUSBRASIL, 27 de fevereiro de 2020. Disponível em: <https://www.jusbrasil.com.br/jurisprudencia/?ref=navbar>. Acesso em: 27 de fevereiro de 2020.

LIMA, Gustavo André Mateus Vaz de et al. As operações de pontos de entrega voluntária como solução de gestão de resíduos sólidos pós-consumo para a economia circular. 2018.

MONTEIL, Cécile. Blockchain and health. In Digital Medicine. Springer, p. 41 - 49, 2019.

MOREIRA, Rodrigo. Investigação preliminar sobre o blockchain e os smart contracts. Revista de Direito e as Novas Tecnologias, v. 3, 2019.

OLIVEIRA, Harlei Rafael; CARDOSO, Ciro Portella. A (I) legalidade das provas obtidas a partir de aplicativos em processo judicial. Plataforma de Submissão de Trabalhos e Anais de Eventos da Unicruz, p. 5, 2019.

ORAM, Andy. Peer-to-Peer: Harnessing the Power of Disruptive Technologies. O'Reilly Media, p. 8, 2001.

ORIGINALMY, 27 de fevereiro de 2020. Disponível em: 〈https://originalmy.com/pacweb>. Acesso em: 27 de fevereiro de 2020. 
SALLES, Marcos Huet Nioac de. A reinvenção do papel do cartório de imóveis na era da tecnologia blockchain: uma investigação exploratória. 2019. Tese de Doutorado.

SILVA, Luan Rodrigues et al. Logística reversa de latas de tinta utilizando Blockchain. 2019.

SOARES, Sílvia Simões. Aspectos jurídicos do compartilhamento de arquivos MP3 P2P via Internet: a experiência do Napster e as novas tendências da legislação de copyright dos Estados Unidos. Direito e Internet: aspectos jurídicos relevantes, v. 2, p. 29, 2008.

TANENBAUM, Andrew S.; STEEN, Maarten Van. Sistemas distribuídos: princípios e paradigmas. Pearson Education do Brasil, 2007.

ULRICH, Fernando. Bitcoin: a moeda na era digital. São Paulo: Instituto Ludwig Von Mises Brasil, p. 18 - 19, 2014.

Data de Submissão: 06/08/2020

Data de Aceite: 18/09/2020 